\title{
THE DOMAIN SPACES OF QUASILOGARITHMIC OPERATORS
}

\author{
M. CWIKEL, B. JAWERTH AND M. MILMAN
}

\begin{abstract}
The construction of intermediate Banach spaces in interpolation theory and the study of commutator inequalities in this context are closely related to certain nonlinear operators $\Omega$. Here an explicit characterization of the domain spaces of these operators $\Omega$ is obtained, and the characterization is related to logarithmic Sobolev inequalities.
\end{abstract}

\section{INTRODUCTION}

In [15] Rochberg and Weiss initiated the study of second order estimates for the complex method of interpolation. In [10] a similar analysis was carried out for the real method of interpolation.

Real interpolation spaces are constructed by means of finding efficient ways of decomposing functions into pieces which can then be studied separately. Let $\bar{A}$, $\bar{B}$, be couples of Banach spaces and let $T: \bar{A} \rightarrow \bar{B}$ be a bounded linear operator. The optimal decompositions can be done before and after application of $T$. This leads to considering operators $\Omega$, which we shall term "quasilogarithmic operators", related to the decomposition process (see $\S 2$ for precise definitions). The main theoretical results of [10] are that the commutator of $T$ and $\Omega$ is bounded on interpolation spaces and that $T$ is bounded on the domain of definition of $\Omega$.

This paper is a contribution to the study of the relationship between the quasilogarithmic operators generated by the $K$ and $J$ methods of interpolation and its implications for the study of the structure of the domains of definition of $\Omega$. In fact, in (2.8) we show that there exist optimal $J$ and $K$ decompositions such that

$$
\Omega_{K}=-\Omega_{J} .
$$

This suggest that the domain spaces $\Omega_{\theta, q}(\bar{A})$ can be obtained as real interpolation spaces similar to the standard spaces $\bar{A}_{\theta, q}$, but with the usual "weight" function $t^{-\theta}$ replaced by $\omega_{\theta}(t)=t^{-\theta}(1+|\log t|)$. For a couple $\bar{A}$ we denote

Received by the editors February 3, 1988.

1980 Mathematics Subject Classification (1985 Revision). Primary 46M35, 46E30.

Part of this research was carried out while two authors (M. C. and M. M.) were at the Institute for Advanced Study, where the first author was supported by NSF Grant MCS-8108814(a04).

The second author was supported in part by NSF Grant DMS-8604528. 
these spaces by $\bar{A}_{\omega_{\theta}, q}$. Using this notation, the question suggested by $(1.1)$ is whether the equality

$$
\Omega_{\theta, q}(\bar{A})=\bar{A}_{\omega_{\theta}, q}
$$

holds for all couples of Banach spaces.

In $\S 2$ we provide a proof of (1.2) using (1.1) and a retract argument. This result clarifies some of the results of [15] and [10] and provides an efficient way of computing the spaces $\Omega_{\theta, q}(\bar{A})$ in many concrete instances (cf. $\S 3$ ).

As will be apparent from our proof, the validity of (1.2) depends ultimately on a "mixed reiteration theorem." In fact, $\Omega_{\theta, q}$ is an interpolation functor, and our argument reduces the proof of (1.2) to checking its validity for a couple of $L^{p}$ spaces. We briefly discuss further aspects of this point of view in $\S 4$.

The papers [15] and [10] contain a number of applications of commutator inequalities to other areas of analysis. In $\S 3$ we discuss the relationship between commutator inequalities and logarithmic Sobolev inequalities, and we compare the space $\Omega_{\theta, q}(\bar{A})$ with a variant of the complex method of interpolation.

\section{COMMUTATOR INEQUALITIES AND QUASILOGARITHMIC OPERATORS}

We start by recalling the relevant definitions and results from [10].

Let $\bar{A}=\left(A_{0}, A_{1}\right)$ be a Banach couple. Given $a \in \Sigma(\bar{A})$ and $c>1$, the decomposition $a=a_{0}(t)+a_{1}(t), t>0$, is c-almost optimal if

$$
K(t, a, \bar{A}) \leq\left\|a_{0}(t)\right\|_{A_{0}}+t\left\|a_{1}(t)\right\|_{A_{1}} \leq c K(t, a, \bar{A})
$$

for $t>0$. (In what follows the constant $c$ will be fixed during the discussion, and its value will be unimportant for our purposes.)

An almost optimal projection is a map defined by taking $D(t) a=D_{K}(t, \bar{A}) a=$ $a_{0}(t)$ for some almost optimal decomposition. $D$ is in general nonlinear, and $D(t): \Sigma(\bar{A}) \rightarrow A_{0}$. It is always possible to choose almost optimal decompositions such that $a_{i}(t), i=0,1$, is a continuous $A_{i}$-valued function of $t$ (e.g. simply specify $a_{i}\left(2^{n}\right)$ for each integer $n$ and extend linearly on each interval $\left.\left(2^{n}, 2^{n+1}\right)\right)$. In such a case we shall say that the $c$-almost optimal decomposition and the associated almost optimal projection are smooth.

Let $\bar{A}, \bar{B}$ be Banach couples, and let $T$ be a bounded linear operator, $T: \bar{A} \rightarrow \bar{B}$. The local commutator $[T, D(t)]$ is defined by

$$
[T, D(t)] a=T D(t, \bar{A}) a-D(t, \bar{B}) T a, \quad a \in \Sigma(\bar{A}), t>0 .
$$

The commutator operator $[T, \Omega]$ is then defined by

$$
[T, \Omega] a=\int_{o}^{\infty}[T, D(t)] a \frac{d t}{t} .
$$

To give the notation $[T, \Omega]$ an interpretation corresponding to the commutator 
of two operators in the usual sense we introduce

(2.2) Definition. Let $\bar{A}$ be a Banach couple and let $D(t), t>0$, be a smooth almost optimal projection. The operator $\Omega=\Omega_{\bar{A}, K}$ is defined by

$$
\Omega a=\Omega_{\bar{A}, K} a=\int_{0}^{1} D(t) a \frac{d t}{t}-\int_{1}^{\infty}(a-D(t) a) \frac{d t}{t} .
$$

Then the formula (2.1) may be written as

$$
[T, \Omega] a=T \Omega_{\bar{A}} a-\Omega_{\bar{B}} T a .
$$

Formally $\Omega$ is a renormalization of the divergent integral $\int_{0}^{\infty} D(t) a \frac{d t}{t}$. For example, for the couple $\bar{A}=\left(L^{1}, L^{\infty}\right)$,

$$
\Omega f(x)=f(x)\left|\log \lambda_{f}(|f(x)|)\right|
$$

where $\lambda_{f}(t)=|\{x:|f(x)| \geq t\}|$ is the left-continuous distribution function of $f$. This and other examples are given in [10]. In the light of examples like this one, and also for reasons which will be apparent later, we shall refer to $\Omega$ as a "quasilogarithmic operator."

Let us also point out that if a different almost optimal projection $\widetilde{D}(t)$ is chosen, then the corresponding quasilogarithmic operator $\widetilde{\Omega}$ will be, in general, different from $\Omega$. However, for $0<\theta<1,1 \leq q \leq \infty, \Omega-\widetilde{\Omega}$ is a bounded map of $\bar{A}_{\theta, q}$ to itself. This is in fact a simple consequence of the commutator theorem (cf. (2.5) and [10]). This justifies the following definition:

For $0<q \leq \infty, 0<\theta<1$, let

$$
\Omega_{\theta, q}(\bar{A})=\left\{a \in \bar{A}_{\theta, q}: \Omega a \in \bar{A}_{\theta, q}\right\}
$$

with the quasi-norm

$$
\|a\|_{\Omega_{\theta, q}(\bar{A})}=\|a\|_{\bar{A}_{\theta, q}}+\|\Omega a\|_{\bar{A}_{\theta, q}} .
$$

Let $\bar{A}, \bar{B}$ be Banach couples, and let $T: \bar{A} \rightarrow \bar{B}$ be a bounded linear operator. Then (cf. [10])

$$
\begin{aligned}
& {[T, \Omega]: \bar{A}_{\theta, q} \rightarrow \bar{B}_{\theta, q}, \quad 0<\theta<1,0<q \leq \infty,} \\
& T: \Omega_{\theta, q}(\bar{A}) \rightarrow \Omega_{\theta, q}(\bar{B}), \quad 0<\theta<1,0<q \leq \infty .
\end{aligned}
$$

Similarly, as in [10], one can define the corresponding quasilogarithmic operators for the $J$ method. Given $a \in \Sigma(\bar{A}), 0<\theta<1,0<q \leq \infty$, we shall say that $D_{J}^{\theta, q} a=u$ defines an almost optimal decomposition for the $J$ method if $u:(0, \infty) \rightarrow \Delta(\bar{A})$ is such that

$$
\begin{gathered}
\int_{0}^{\infty} u(t) \frac{d t}{t}=a \text { in } \Sigma(\bar{A}) \\
\left(\int^{\infty}\left(J(t, u(t), \bar{A}) t^{-\theta}\right)^{q} \frac{d t}{t}\right)^{1 / q} \leq c\|a\|_{\bar{A}_{\theta, q . J}} .
\end{gathered}
$$


The associated quasilogarithmic operator is defined by

$$
\Omega_{\theta, q, J} a=\int_{0}^{\infty} u(t) \log t \frac{d t}{t} .
$$

In [10] it is shown that (2.5) also holds when the quasilogarithmic operators $\Omega_{\bar{A}, K}$ and $\Omega_{\bar{B}, K}$ are replaced by their $\Omega_{\theta, q, J}$ analogues.

Our first result clarifies the relationship between the quasilogarithmic operators generated by the $K$ and $J$ methods (cf. [10]):

(2.8) Theorem. Let $\bar{A}$ be a Banach couple and let $a \in \Sigma^{0}(\bar{A})$. Then there exists a decomposition for the $J$ method $a=\int_{0}^{\infty} u(t) \frac{d t}{t}$ which is almost optimal for all choices of $\theta \in(0,1), q \in(0, \infty]$. Furthermore, there exists an almost optimal decomposition for the $K$ method such that the associated quasilogarithmic operators $\Omega_{J}, \Omega_{K}$ satisfy

$$
\Omega_{J} a=-\Omega_{K} a .
$$

Proof. Let $a \in \Sigma(\bar{A})$. By the fundamental lemma of interpolation theory (cf. $[2,4])$ we can find a representation $a=\int_{0}^{\infty} u(s) \frac{d s}{s}$ such that an almost optimal decomposition for the $K$ method is given by

$$
a=\int_{0}^{t} u(s) \frac{d s}{s}+\int_{t}^{\infty} u(s) \frac{d s}{s}
$$

and

$$
\begin{aligned}
K(t, a, \bar{A}) & \leq \int_{0}^{\infty} \min \left(1, \frac{t}{s}\right) J(s, u(s), \bar{A}) \frac{d s}{s} \\
& \leq c \int_{0}^{\infty} \min \left(1, \frac{t}{s}\right) K(s, a, \bar{A}) \frac{d s}{s}
\end{aligned}
$$

for $t>0$. This (and the equivalence of $\bar{A}_{\theta, q, K}$ and $\bar{A}_{\theta, q, J}$ ) implies that the decomposition $D_{J} a(t)=u(t)$ is an almost optimal decomposition of $a$, for all choices of $\theta$ and $q$. Also, $D_{K}(t) a=\int_{0}^{t} u(s) \frac{d s}{s}$ is an almost optimal projection.

Now we calculate

$$
\begin{aligned}
\Omega_{K} a & =\int_{0}^{1} D_{K}(t) a \frac{d t}{t}-\int_{1}^{\infty}\left(a-D_{K}(t) a\right) \frac{d t}{t} \\
& =\int_{0}^{1} \int_{0}^{t} u(s) \frac{d s}{s} \frac{d t}{t}-\int_{1}^{\infty} \int_{t}^{\infty} u(s) \frac{d s}{s} \frac{d t}{t} \\
& =\int_{0}^{1}(-\log s) u(s) \frac{d s}{s}-\int_{1}^{\infty} \int_{1}^{s} \frac{d t}{t} u(s) \frac{d s}{s} \\
& =-\int_{0}^{\infty} u(s) \log s \frac{d s}{s}=-\Omega_{J} a,
\end{aligned}
$$

and this completes the proof.

It follows from (2.9) that the domain spaces $\Omega_{\theta, q, J}(\bar{A})$ coincide with the corresponding space $\Omega_{\theta, q, K}(\bar{A})$ (cf. (2.4)). 
We next consider the relationship between the spaces $\Omega_{\theta, q}(\bar{A})$ and the interpolation spaces obtained by replacing the weights $t^{-\theta}$ by

$$
\omega_{\theta}(t)=(1+|\log t|) t^{-\theta} \text {. }
$$

Given a Banach couple $\bar{A}, 0<\theta<1,0<q \leq \infty$, we define

$$
\begin{aligned}
\bar{A}_{\omega_{\theta}, q, K}=\left\{a \in \Sigma(\bar{A}):\|a\|_{\bar{A}_{\omega_{\theta}, q, K}}\right. & \\
= & \left.\left(\int_{0}^{\infty}\left(\omega_{\theta}(t) K(t, a, \bar{A})\right)^{q} \frac{d t}{t}\right)^{1 / q}<\infty\right\} .
\end{aligned}
$$

In a similar fashion one defines the corresponding $\bar{A}_{\omega_{\theta}, q, J}$ spaces and one verifies, using the fundamental lemma and a suitable form of Hardy's inequalities, that

$$
\bar{A}_{\omega_{\theta}, q, K} \simeq \bar{A}_{\omega_{\theta}, q, J}, \quad 0<\theta<1,0<q \leq \infty .
$$

(For even more general results see [9].)

Our main result is given by

$$
\text { Theorem. } \Omega_{\theta, q}(\bar{A})=\bar{A}_{\omega_{\theta}, q, J}, 0<\theta<1,0<q \leq \infty \text {. }
$$

For the proof we shall need the following two lemmas. The first is concerned with the stability of the spaces $\Omega_{\theta, q}(\bar{A})$ under iteration, while the second describes the stability of $\Omega$ under partial retracts.

(2.13) Lemma. Let $\bar{A}$ be a couple of Banach spaces, $0<\theta_{0}<\theta_{1}, \quad 1 \leq$ $q_{0}, q, q_{1} \leq \infty, 0<\theta, \lambda<1$. If $\bar{A} \overline{\theta, q}=\left(\bar{A}_{\theta_{0}, q_{0}}, \bar{A}_{\theta_{1}, q_{1}}\right),(1-\lambda) \theta_{0}+\lambda \theta_{1}=\theta$, then

$$
\Omega_{\lambda, q}(\bar{A} \overline{\theta, q})=\Omega_{\theta, q}(\bar{A}) .
$$

Proof. By Holmstedt's proof of the reiteration theorem (cf. $[1,10]$ ),

$$
D_{K}(t, \bar{A} \overline{\theta q})(a)=D_{K}\left(t^{1 / \alpha}, \bar{A}\right)(a)
$$

where $\alpha=\theta_{1}-\theta_{0}$. Therefore

$$
\alpha \cdot \Omega_{\overline{A_{\overline{\theta, q}}}}=\Omega_{\bar{A}}
$$

and (2.14) follows.

Let us recall the notion of partial retract. Given two couples $\bar{A}, \bar{B}$, we say that $\bar{A}$ is a partial retract of $\bar{B}$ if for each $x \in \Sigma(\bar{A})$ there are two bounded linear operators $\mathscr{I}: \bar{A} \rightarrow \bar{B}$ and $\mathscr{P}: \bar{B} \rightarrow \bar{A}$ such that $\mathscr{P} \mathscr{F} x=x$ and the norms of $\mathscr{I}$ and $\mathscr{P}$ are independent of $x$. This notion apparently goes back to Peetre [13] (where the terminology is slightly different).

(2.16) Lemma. Let $\bar{A}$ be a partial retract of $\bar{B}$, and let $0<\theta<1,0<q \leq \infty$. If $\Omega_{\theta, q}(\bar{B}) \subseteq \bar{B}_{\omega_{\theta}, q}$, then we also have $\Omega_{\theta, q}(\bar{A}) \subseteq \bar{A}_{\omega_{\theta}, q}$. 
Proof. Let $a \in \Omega_{\theta, q}(\bar{A})$. Let $\mathscr{I}: \bar{A} \rightarrow \bar{B}, \mathscr{P}: \bar{B} \rightarrow \bar{A}$ be such that $\mathscr{P} \mathscr{f} a=a$. By the commutator theorem

$$
[\Omega, \mathscr{J}]: \bar{A}_{\theta, q} \rightarrow \bar{B}_{\theta, q} .
$$

Therefore, the formula

$$
[\Omega, \mathscr{I}] a=\mathscr{I} \Omega_{A} a-\Omega_{B} \mathscr{I} a
$$

shows that $\mathscr{F} a \in \Omega_{\theta, q}(\bar{B}) \subseteq \bar{B}_{\omega_{\theta}, q}$.

By interpolation,

$$
\mathscr{P}: \bar{B}_{\omega_{\theta}, q} \rightarrow \bar{A}_{\omega_{\theta}, q}
$$

and so $\mathscr{P}(\mathscr{I} a)=a \in \bar{A}_{\omega_{\theta}, q}$, as required.

We now return to the proof of (2.12):

Proof. Let $a \in \bar{A}_{\omega_{\theta}, q, J}$. As in the proof of (2.8) there exists a representation $a=\int_{0}^{\infty} r(s) \frac{d s}{s}$ such that

$$
\Omega_{K} a=-\int_{0}^{\infty} \log s r(s) \frac{d s}{s}
$$

and

$$
\left(\int_{0}^{\infty}\left(\omega_{\theta}(t) J(t, r(t), \bar{A})\right)^{q} \frac{d t}{t}\right)^{1 / q} \leq c\|a\|_{\bar{A}_{\omega_{\theta}, q . J}} .
$$

Let $u(s)=(\log s) r(s)$. Then $\Omega_{K} a=\int_{0}^{\infty} u(s) \frac{d s}{s}$ and

$$
\begin{aligned}
\left.\left(\int_{0}^{\infty}\left(t^{-\theta} J(t, u](t), \bar{A}\right)\right)^{q} \frac{d t}{t}\right)^{1 / q} & \leq c\left(\int_{0}^{\infty}\left(\omega_{\theta}(t) J(t, r(t), \bar{A})\right)^{q} \frac{d t}{t}\right)^{1 / q} \\
& \leq c\|a\|_{\bar{A}_{\omega_{\theta} . q . J}} .
\end{aligned}
$$

Consequently, $\Omega a \in \bar{A}_{\theta, q, J}$ and $\|\Omega a\|_{\bar{A}_{\theta, q, J}} \leq c\|a\|_{\bar{A}_{\omega_{\theta}, q . J}}$.

Conversely, let us show that $\Omega_{\theta, q}(\bar{A}) \subseteq \bar{A}_{\omega_{\theta}, q}$. Observe that by the reiteration formula (2.14) we only need to show that $\Omega_{\lambda, q}\left(\bar{A}_{\theta_{0}, q_{0}}, \bar{A}_{\theta_{1}, q_{1}}\right) \subseteq \bar{A}_{\omega_{\theta}, q}$, for whichever particular choice of $q_{0}$ and $q_{1}$ in $[1, \infty]$ which we find most convenient, and where the other indices satisfy the conditions of Lemma (2.13). Now, it was proved in [5] that the couple $\left(\bar{A}_{\theta_{0}, q_{0}}, \bar{A}_{\theta_{1}, q_{1}}\right)$ is a partial retract of the couple $\left(l^{q_{0}}\left(2^{-n \theta_{0}}\right), l^{q_{1}}\left(2^{-n \theta_{1}}\right)\right)$. Moreover, we shall verify in a moment that

$$
\Omega_{\dot{\lambda}, q}\left(l^{q_{0}}\left(2^{-n \theta_{0}}\right), l^{q_{1}}\left(2^{-n \theta_{1}}\right)\right) \subseteq\left(l^{q_{0}}\left(2^{-n \theta_{0}}\right), l^{q_{1}}\left(2^{-n \theta_{1}}\right)\right)_{\omega_{\lambda}, q} .
$$

Hence, by Lemma (2.16),

$$
\Omega_{\lambda, q}\left(\bar{A}_{\theta_{0}, q_{0}}, \bar{A}_{\theta_{1}, q_{1}}\right) \subset\left(\bar{A}_{\theta_{0}, q_{0}}, \bar{A}_{\theta_{1}, q_{1}}\right)_{\omega_{i}, q}=\bar{A}_{\omega_{\theta}, q},
$$

where the last equality follows from the reiteration theorem of [8]. Thus we have shown that the desired result will follow from (2.19). In order to verify (2.19) we argue that by successive partial retracts (cf. example (3.7) below) it is enough to verify (2.19) for the couple $\left(l^{q_{0}}, l^{q_{1}}\right)$. The required result is then contained in the following. 
(2.20) Lemma. Let $\left(L^{q_{0}}(X), L^{q_{1}}(X)\right), 1 \leq q_{0} \neq q_{1} \leq \infty$, be a couple of $L^{q}$ spaces on a measure space $X$. Then

$$
\Omega_{\theta, q}\left(L^{q_{0}}(X), L^{q_{1}}(X)\right) \subset\left(L^{q_{0}}(X), L^{q_{1}}(X)\right)_{\omega_{\theta}, q}
$$

for $0<\theta<1,0<q \leq \infty$.

Proof. We first observe that by reiteration $((2.13)$ and (2.15)) it is enough to consider the case $q_{0}=1$ and $q_{1}=\infty$. Then

$$
\Omega a(x)=a(x) \mid \log \lambda_{a}(|a(x)|),
$$

and $K\left(t, a ; L^{1}, L^{\infty}\right)=\int_{0}^{t} a^{*}(s) d s$. The norm of an element $a$ in the space on the left-hand side is equivalent to

$$
\left(\int_{0}^{\infty}\left[t^{-\theta} K\left(t,|a|+|\Omega a| ; L^{1}, L^{\infty}\right)\right]^{q} \frac{d t}{t}\right)^{1 / q} .
$$

We claim that

$$
K(t,|a|+|\Omega a|) \geq a^{*}(t)(1+|\log t|) t .
$$

It would then follow that the above integral is greater than

$$
\left(\int_{0}^{\infty}\left[\omega_{\theta}(t) t a^{*}(t)\right]^{q} \frac{d t}{t}\right)^{1 / q}
$$

which in turn is equivalent to the norm of $a$ in the space on the right-hand side.

It thus remains to show (2.21) and that is very easy: Since

$$
K\left(t, a ; L^{1}, L^{\infty}\right)=\int_{0}^{t} a^{*}(s) d s=\sup _{|E| \leq t} \int_{E}|a|,
$$

we see that $K(t,|a|+|\Omega a|) \geq \int_{E}|a|+|\Omega a|$ where $E \subset\left\{x \in X:|a(x)| \geq a^{*}(t)\right\}$ and $|E|=t$. Using that $|a|+|\Omega a| \geq a^{*}(t)(1+|\log t|)$ on $E$, and that $|E|=t$, (2.21) follows.

\section{EXAMPLES AND APPLICATIONS}

In this section we consider several examples and applications of the results of $\S 2$. In particular, we relate the quasilogarithmic norm inequalities with the so-called logarithmic Sobolev inequalities and with the complex method of ințerpolation.

Let $\bar{A}=\left(A_{0}, A_{1}\right)$ be a couple of Banach spaces such that $A_{0} \supseteq A_{1}$. Let $0<\theta_{0} \leq \theta \leq \theta_{1}<1,0<q \leq \infty$. Then using Hölder's inequality, we have for $a \in \Delta(\bar{A})$,

$$
\|a\|_{\bar{A}_{\theta, q, K}} \leq\|a\|_{\bar{A}_{\theta_{0}, q, K}}^{\left(\theta_{1}-\theta\right) /\left(\theta_{1}-\theta_{0}\right)}\|a\|_{\bar{A}_{\theta_{1}, q, K}}^{\left(\theta-\theta_{0}\right) /\left(\theta_{1}-\theta_{0}\right)}
$$


Observe that both sides of (3.1) coincide for $\theta=\theta_{0}$. Therefore, differentiating both sides of (3.1) at $\theta=\theta_{0}$ we obtain

$$
\left.\frac{d}{d \theta}\left(\|a\|_{\bar{A}_{\theta, q, K}}\right)\right|_{\theta=\theta_{0}} \leq\|a\|_{\bar{A}_{\theta_{0}, q, K}} \log \left(\frac{\|a\|_{\bar{A}_{\theta_{1}, q, K}}}{\|a\|_{\bar{A}_{\theta_{0}, q, K}}}\right)^{1 /\left(\theta_{1}-\theta_{0}\right)} .
$$

Thus

$$
\|a\|\left\|_{A_{\theta_{0}, q, K}}^{1-q}\right\| a\left\|_{A_{\omega_{\theta_{0}, q, q, K}}^{q}} \leq\right\| a \|_{\bar{A}_{\theta_{0}, q, K}} \log \left(\frac{\|a\|_{\bar{A}_{\theta_{1}, q, K}}}{\|a\|_{\bar{A}_{\theta_{0}, q, K}}}\right)^{1 /\left(\theta_{1}-\theta_{0}\right)}
$$

where $\omega_{\theta_{0}, q}(t)=t^{-\theta_{0}}\left(\log \frac{1}{t}\right)^{1 / q}, 0<t<1$, and where $\bar{A}_{\omega, q, K}$ for any positive weight function $\omega(t)$ denotes another by now standard generalization of the space $\bar{A}_{\theta, q, K}$.

In particular, if $q=1$, we obtain, using (2.12),

$$
\|\Omega a\|_{\bar{A}_{\theta_{0}, 1}} \leq c\|a\|_{\bar{A}_{\theta_{0}, 1}} \log \left(\frac{\|a\|_{\bar{A}_{\theta_{1}, 1}}}{\|a\|_{\bar{A}_{\theta_{0}, 1}}}\right)^{1 /\left(\theta_{1}-\theta_{0}\right)} .
$$

Inequalities of this form appear in the theory of logarithmic Sobolev inequalities (cf. [7]). With appropriate choices, one can interpret (3.3) as "moment inequalities" (cf. [14] and our next example).

(3.4) Example. Let $X$ be a Banach space and let $\Lambda$ be a linear closed operator with domain of definition $D(\Lambda) \subseteq X$ and dense. Assume, moreover, that $\Lambda$ is positive in the sense that there exists a constant $C>0$ such that $\left\|(\Lambda+t I)^{-1}\right\| \leq$ $C /(1+t)$ for $t \geq 0$. Then (cf. [10,17]) one can compute $D_{K}$ for the couple $(X, D(\Lambda))$ and obtain $\Omega_{K}=C \log (I+\Lambda)$. In this case (3.3) can be interpreted as a moment inequality of the type considered in [14].

Next we consider the case of Sobolev spaces.

(3.5) Example. Let $R$ be a minimally smooth domain, $R \subseteq \mathbf{R}^{n},|R|<\infty$. For $k \in N, 1 \leq p \leq \infty$, consider the Sobolev spaces $W_{p}^{k}(R)=\left\{f: D^{\alpha} f \in\right.$ $\left.L^{p}(R), \forall|\alpha| \leq k\right\}$. Let $\bar{A}=\left(W_{1}^{k}, W_{\infty}^{k}\right)$. Then (cf. [3]),

$$
K(t, f, \bar{A}) \approx \sum_{|\alpha| \leq k} \int_{0}^{t}\left(D^{\alpha} f\right)^{*}(s) d s, \quad 0<|t|<|R| .
$$

It follows that

$$
\Omega_{\theta, q}(\bar{A})=\left\{f: D^{\alpha} f \log \left|D^{\alpha} f\right| \in L\left(\frac{1}{1-\theta}, q\right), \forall|\alpha| \leq k\right\} .
$$

(3.6) Example. The notion of a partial retract plays an important role in $\S 2$. Here we wish to point out that if $\bar{A}$ is a retract of $\bar{B}$ (i.e., there exist operators $\mathscr{I}: \bar{A} \rightarrow \bar{B}, \mathscr{P}: \bar{B} \rightarrow \bar{A}$ such that $\mathscr{P} \mathscr{F}=\mathrm{id}_{\bar{A}}$ ), then there is a simple formula relating $\Omega_{\bar{A}}$ and $\Omega_{\bar{B}}$, namely $\Omega_{\bar{A}}=\mathscr{P} \Omega_{\bar{B}} \mathscr{I}^{A}$. This allows us to simplify many computations. 
An interesting case where (3.6) applies is given by couples of weighted $L^{p}$ spaces.

(3.7) Example. Let $1 \leq p_{0} \neq p_{1} \leq \infty$. We consider a couple of weighted $L^{p}$ spaces on a measure space $X$. Given positive functions $w_{i}, i=0,1$, on $X$, we define the weighted spaces $L_{w_{i}}^{p_{i}}$ by the norm $\left(\int\left(|f| w_{i}\right)^{p_{i}} d x\right)^{1 / p_{i}}$. Let $\bar{A}$ be the couple $\left(L_{w_{0}}^{p_{0}}, L_{w_{1}}^{p_{1}}\right)$; then $\bar{A}$ is the retract of the couple $\bar{B}=\left(L^{p_{0}}(\sigma(x) d x)\right.$, $\left.L^{p_{1}}(\sigma(x) d x)\right)$ where the measure

$$
\sigma(x) d x=w_{1}^{p_{0} p_{1} /\left(p_{0}-p_{1}\right)} w_{0}^{\left(-p_{0} p_{1}\right) /\left(p_{0}-p_{1}\right)} d x .
$$

In fact, the map $\mathscr{I}: \bar{A} \rightarrow \bar{B}$ is given by

$$
\mathscr{I} f=\left(\frac{w_{0}^{p_{0}}}{w_{1}^{p_{1}}}\right)^{1 /\left(p_{0}-p_{1}\right)} f \text { and } \mathscr{P} f=\left(\frac{w_{1}^{p_{1}}}{w_{0}^{p_{0}}}\right)^{1 /\left(p_{0}-p_{1}\right)} f
$$

(with the obvious interpretations when one of the $p$ 's is infinite). This idea goes back of course to the paper [16] by Stein and Weiss.

To relate the quasilogarithmic operators generated by the real and complex methods we introduce a new method of complex interpolation. A variant of this method was introduced in [6].

(3.8) Definition. Let $\bar{A}=\left(A_{0}, A_{1}\right)$ be a Banach couple. For $0<\theta<1$ we define the interpolation spaces

$$
\operatorname{Der}_{\theta}(\bar{A})=\{a \in \Sigma(\bar{A}): \exists f \in \tilde{\mathscr{F}}(\bar{A}) \text { s.t. } f(\theta)=a\}
$$

where $\tilde{F}(\bar{A})$ is the usual class $\mathscr{F}(\bar{A})$ of Calderón (cf. [1]) with the additional condition that $f^{\prime}(\theta) \in[\bar{A}]_{\theta} \quad$ (= usual complex interpolation space).

Let $0<\theta_{0}<\theta<\theta_{1}, 1 \leq q_{0}, q_{1} \leq 1,0<\eta<1$. Then,

$$
\bar{A}_{\theta, q}=\left[\bar{A}_{\theta_{0}, q_{0}}, \bar{A}_{\theta_{1}, q_{1}}\right]_{\eta} \text {, }
$$

where $\theta=(1-\eta) \theta_{0}+\eta \theta_{1}, \frac{1}{q}=\frac{1-\eta}{q_{0}}+\frac{\eta}{q_{1}}$ (cf. [1] and §4). Let $a \in \bar{A}_{\theta, q}$ and choose an almost optimal $J$ decomposition independent of $(\theta, q), a=$ $\int_{0}^{\infty} u(s) \frac{d s}{s}$. Then we can construct an analytic function $f \in \mathscr{F}\left(\bar{A}_{\theta_{0}, q_{0}}, \bar{A}_{\theta_{1}, q_{1}}\right)$ by letting

$$
f(z)=\int_{0}^{\infty} t^{\gamma(z-\eta)} u(t) \frac{d t}{t}
$$

where $\gamma=\left(\theta-\theta_{0}\right) / \eta$. Clearly, $f(\eta)=a$ and $f^{\prime}(\eta)=\left(\theta-\theta_{0}\right) \Omega_{J} a$. Consequently, we have that

$$
\Omega_{\theta, q}(\bar{A}) \subseteq \operatorname{Der}_{\eta}\left(\bar{A}_{\theta_{0}, q_{0}}, \bar{A}_{\theta_{1}, q_{1}}\right) .
$$

We can also obtain results of type (3.10) under the assumption that $\bar{A}$ has Fourier types $\left(p_{0}, p_{1}\right)$ (cf. [11]). In such a case we get

$$
\Omega_{\theta, p}(\bar{A}) \subseteq \operatorname{Der}_{\theta}(\bar{A}), \quad \frac{1}{p}=\frac{1-\theta}{p_{0}}+\frac{\theta}{p_{1}} .
$$




\section{MiXed Reiteration}

The method of proof of (1.2) is based on the fact that the couple $\left(\bar{A}_{\theta_{0}, q_{0}}\right.$, $\left.\bar{A}_{\theta_{1}, q_{1}}\right)$ is a partial retract of $\left(l^{q_{0}}\left(2^{-n \theta_{0}}\right), l^{q_{1}}\left(2^{-n \theta_{1}}\right)\right)$. A consequence of this fact is the mixed reiteration formula (cf. $[9,22]$ ).

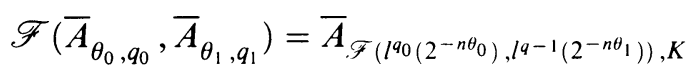

where $\mathscr{F}$ is any interpolation functor, and the space appearing to the right of (4.1) is defined by the condition that

$$
K\left(2^{n}, a, \bar{A}\right) \in \mathscr{F}\left(l^{q_{0}}\left(2^{-n \theta_{0}}\right), l^{q-1}\left(2^{-n \theta_{1}}\right)\right) .
$$

An important example is obtained by letting $\mathscr{F}=[,]_{\theta}$ be the complex method (cf. (3.9) above). Another pertinent example is provided by $\mathscr{F}=\Omega_{\theta, q}$. In fact, by (2.6) $\Omega_{\theta, q}$ is an interpolation functor and therefore (4.1) provides a slightly different route to (1.2).

Let us conclude by observing that (4.1) can be applied to the case $\mathscr{F}=\operatorname{Der}_{\eta}$ thus obtaining some further information related to (3.9):

$$
\operatorname{Der}_{\eta}\left(\bar{A}_{\theta_{0}, q_{0}}, \bar{A}_{\theta_{1}, q_{1}}\right)=\bar{A}_{\operatorname{Der}_{\eta}\left(q _ { 0 } \left(2^{\left.\left.-n \theta_{0}\right), l q_{1}\left(2^{-n \theta_{1}}\right)\right), K}\right.\right.} \cdot
$$

\section{REFERENCES}

1. J. Bergh and J. Löfström, Interpolation spaces: An introduction, Springer-Verlag, Berlin, Heidelberg and New York, 1976.

2. Ju A. Brudnyi and N. Ju Krugljak, Real interpolation functors, Soviet Math. Dokl. 23 (1981), $5-8$.

3. C. P. Calderón and M. Milman, Interpolation of Sobolev spaces. The real method, Indiana Math. J. 32 (1983), 801-808.

4. M. Cwikel, K-divisibility of the K-functional and Calderón couples, Ark. Mat. 22 (1984), $39-62$.

5. __ Monotonicity properties of interpolation spaces. II, Ark. Mat. 19 (1981), 123-136.

6. M. Cwikel, M. Milman and J. Peetre, Complex extrapolation (in preparation).

7. L. Gross, Logarithmic Sobolev inequalities, Amer. J. Math. 97 (1975), 1061-1083.

8. J. Gustafsson, A function parameter in connection with interpolation of Banach spaces, Math. Scand. 42 (1978), 289-305.

9. S. Janson, Minimal and maximal methods of interpolation, J. Funct. Anal. 44 (1981), 50-73.

10. B. Jawerth, R. Rochberg and G. Weiss, Commutator and other second order estimates in real interpolation theory, Ark. Mat. 24 (1986), 191-219.

11. M. Milman, Complex interpolation and geometry of Banach spaces, Ann. Mat. Pura Appl. 86 (1984), 317-328.

12. V. I. Ovcinnikov, The method of orbits in interpolation theory, Math. Rep. 1 (1984), 349-516.

13. J. Peetre, Banach couples, Technical report, Lund, 1971.

14. E. I. Pustylnik, On functions of a positive operator, Math. USSR-Sb. 47 (1984), 27-42.

15. R. Rochberg and G. Weiss, Derivatives of analytic families of Banach spaces, Ann. of Math. 118 (1983), 315-347. 
16. E. M. Stein and G. Weiss, Interpolation of operators with change of measures, Trans. Amer. Math. Soc. 87 (1958), 159-172.

17. H. Triebel, Interpolation theory, function spaces, differential operators, North-Holland, Amsterdam, 1978.

Department of Mathematics, Technion-Israel Institute of Technology, Haifa 32000, ISRAEL (Current address of M. Cwikel)

Department of Mathematics, Washington University, St. Louis, Missouri 63130

Department of Mathematics, Florida Atlantic University, Boca Raton, Florida 33431 (Current address of M. Milman)

Current address (Björn Jawerth): Department of Mathematics, University of South Carolina, Columbia, South Carolina 29208 